\title{
Reconfigured and Notched Tapered Slot UWB Antenna for Cognitive Radio Applications
}

\author{
Tamer Aboufoul, Akram Alomainy, and Clive Parini \\ School of Electronic Engineering and Computer Science, Queen Mary University of London, Mile End Road, London E1 4NS, UK \\ Correspondence should be addressed to Tamer Aboufoul, ta302@eecs.qmul.ac.uk
}

Received 22 February 2012; Accepted 22 October 2012

Academic Editor: Hala A. Elsadek

Copyright (C) 2012 Tamer Aboufoul et al. This is an open access article distributed under the Creative Commons Attribution License, which permits unrestricted use, distribution, and reproduction in any medium, provided the original work is properly cited.

\begin{abstract}
A compact reconfigurable and notched ultra-wideband (UWB) tapered slot antenna (TSA) is presented. The antenna reconfiguration operation principle relies on 2 mechanisms: in the first mechanism a resonator parasitic microstrip line electrically coupled to the TSA is used to notch the TSA at a specific frequency and the second mechanism relies on changing the input impedance matching of the antenna by means of changing the length of a stub line extended from an additional tiny partial ground on the back side of the antenna. The reflection coefficient, radiation patterns, and gain simulations and measurements for the proposed antenna are presented to verify the design concepts featuring a very satisfactory performance. Total efficiency simulations and measurements are also presented to highlight the filtering performance of the reconfigured antenna. When the antenna was reconfigured from the UWB to work into multiple frequency bands, the radiation patterns were still the same and the total peak gain has slightly improved compared to the UWB case. In addition, when the antenna operated in the notched mode, the gain has significantly dropped at the notch frequency. The simplicity and flexibility of the proposed multimode antenna make it a good candidate for future cognitive radio front ends.
\end{abstract}

\section{Introduction}

Reconfigurable UWB antennas can be deployed in cognitive radio (CR) front ends by reconfiguring UWB antenna to work into multiple predefined frequency bands. When the antenna is in the UWB mode, it can be used for sensing the spectrum and when it is operating in the reconfigured modes, the antenna can be used for communication purpose [1-4]. Several designs of UWB antenna with band rejection characteristics have also targeted the cognitive radio applications $[5,6]$. The proposed reconfigurable antenna here is mixing both approaches (e.g., reconfiguration and band rejection in the same antenna design). The reference antenna which will be reconfigured is presented in [7]. This antenna features wide operating bandwidth, very simple and compact structure, ease of fabrication, good radiation patterns over the entire bandwidth, and good time domain performance. In the proposed antenna design, reconfigurability is introduced by employing stubs and changing the electrical length of the stubs by using multiple switches. These stubs are connected to an additional tiny partial ground printed on the back side of the tapered slot antenna (TSA). When the stub is disconnected from the ground it can be used as a parasitic resonator to notch the UWB antenna at specific unwanted frequency. In Section 2, the antenna design is presented and the operation of the antenna is briefly discussed. In Section 3 we show the simulated and measured results for the performance of the antenna. In Section 4 we briefly discuss the applicability of this new design in cognitive radio applications. Finally, conclusions are drawn and presented in Section 5.

\section{Antenna Design and Operation}

Figure 1 shows the geometry of the proposed reconfigured UWB TSA antenna. The proposed antenna substrate is a Taconic board with thickness of $1.52 \mathrm{~mm}$, relative dielectric constant of 3 , and loss tangent of 0.003 . The width of the antenna is $16 \mathrm{~mm}\left(0.28 \lambda_{g}\right)$ and the length of the antenna is $27 \mathrm{~mm}\left(0.47 \lambda_{g}\right)$ where $\lambda_{g}$ is the guided wavelength at $3 \mathrm{GHz}$. On the back side of the substrate, a small partial ground $(12 \mathrm{~mm} \times 1 \mathrm{~mm})$ is printed and there is a stub 


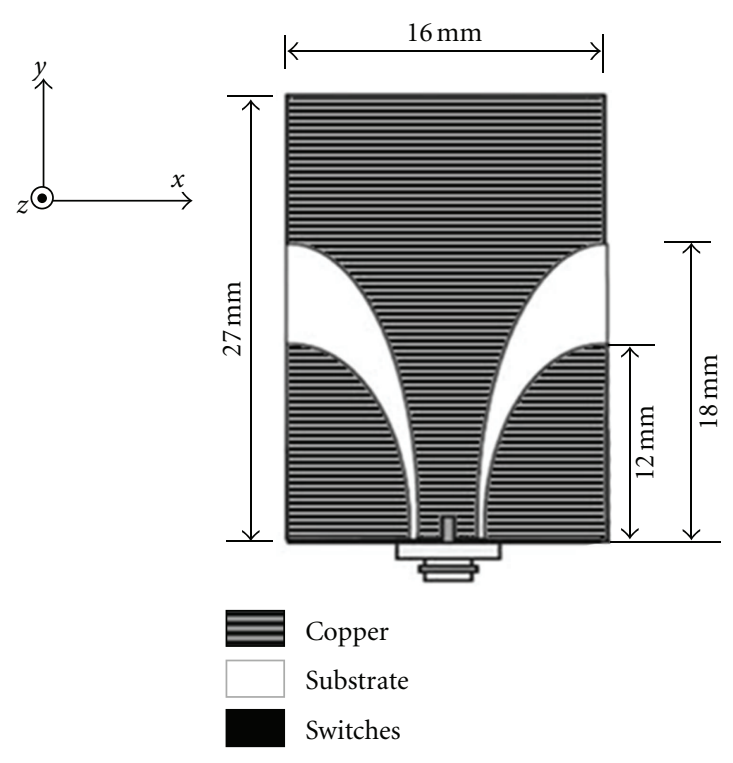

(a)

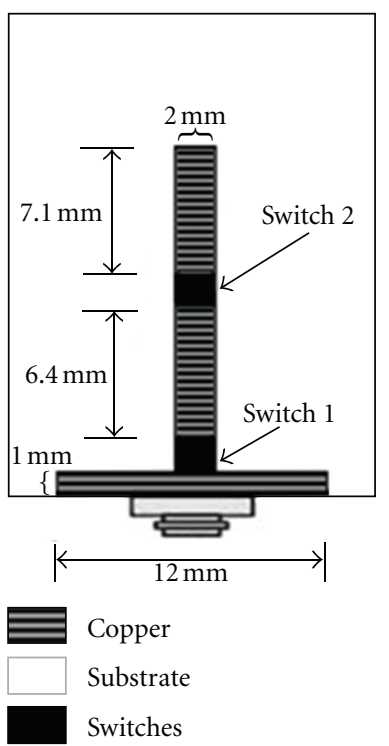

(b)

Figure 1: Geometry of the reconfigured tapered slot antenna: (a) top view, (b) bottom view.

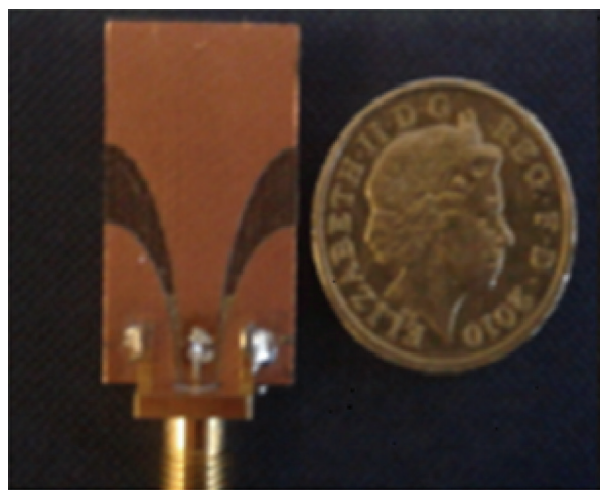

(a)

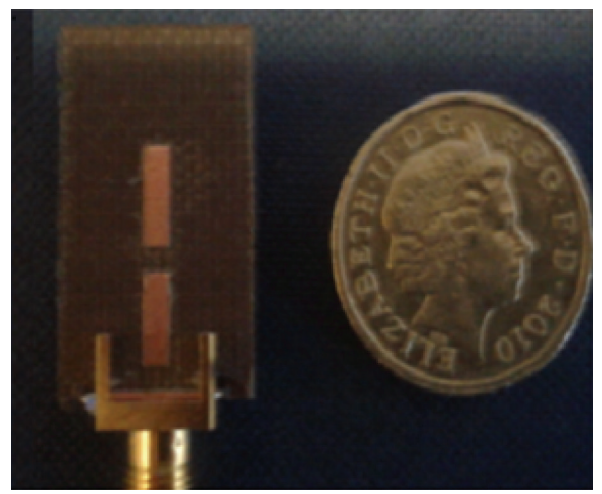

(b)

Figure 2: Antenna prototype: (a) top view, (b) bottom view.

connected to this ground by switch 1 . By changing the length of the stub, we can change the input impedance matching of the antenna to work in predefined frequency bands. The length of the ground stub is determined from circuit analysis using ADS [8]. When the stub length is $8.4 \mathrm{~mm}$, the antenna should be working between 3 and $4 \mathrm{GHz}$. When the stub length increases to $17.5 \mathrm{~mm}$ when both switches 1 and 2 are $\mathrm{ON}$, the frequency band of operation will change to 4.5$5.5 \mathrm{GHz}$. When the ground stub is not connected to the partial ground (e.g., switch 1 is OFF and switch 2 is ON), the stub will act as a parasitic element electrically coupled to the TSA. The length of the parasitic resonator determines the notch frequency and if we want to notch the upper band of WLAN at $5.8 \mathrm{GHz}$, the length of the resonator should be around $15 \mathrm{~mm}$ which is equal to $\lambda_{g} / 2$ where $\lambda_{g}$ is the guided wavelength at $5.8 \mathrm{GHz}$. Full UWB operation without any notches can be achieved if we can kill the resonance coupling of the parasitic element (e.g., the total length of the parasitic
TABLE 1: Operation modes of the antenna.

\begin{tabular}{lcc}
\hline Switch 1 & \multicolumn{2}{c}{ Switch 2 } \\
& ON & OFF \\
\hline ON & Band II & Band I \\
OFF & Notched-UWB & UWB \\
\hline
\end{tabular}

resonator is changed when switch 2 is OFF); consequently, it cannot support the resonating currents and no notch occurs at $5.8 \mathrm{GHz}$. The operation modes of the antenna for different switch states are summarized in Table 1.

A prototype antenna was fabricated to verify the performance. Figure 2 shows the proposed prototype antenna. RF switches used in this prototype are realized as metal pads with dimensions $2 \mathrm{~mm} \times 2 \mathrm{~mm}$. Although this model is ideal, it gives a good approximation for the commercial PIN diode switches. 


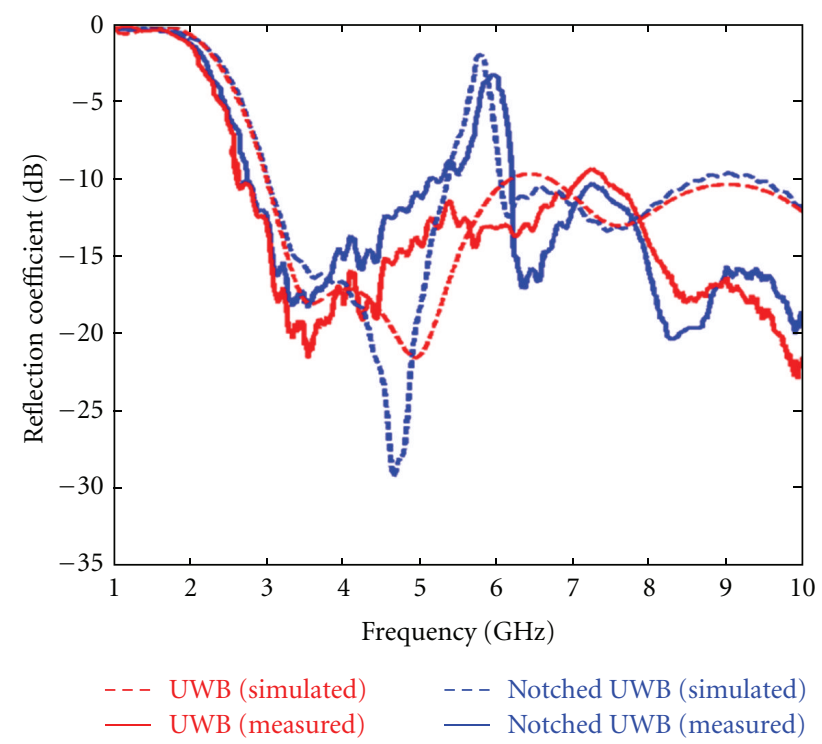

FIGURE 3: Simulated (dashed lines) and measured (solid lines) reflection coefficient curves when antenna is in UWB mode and in the notched UWB mode.

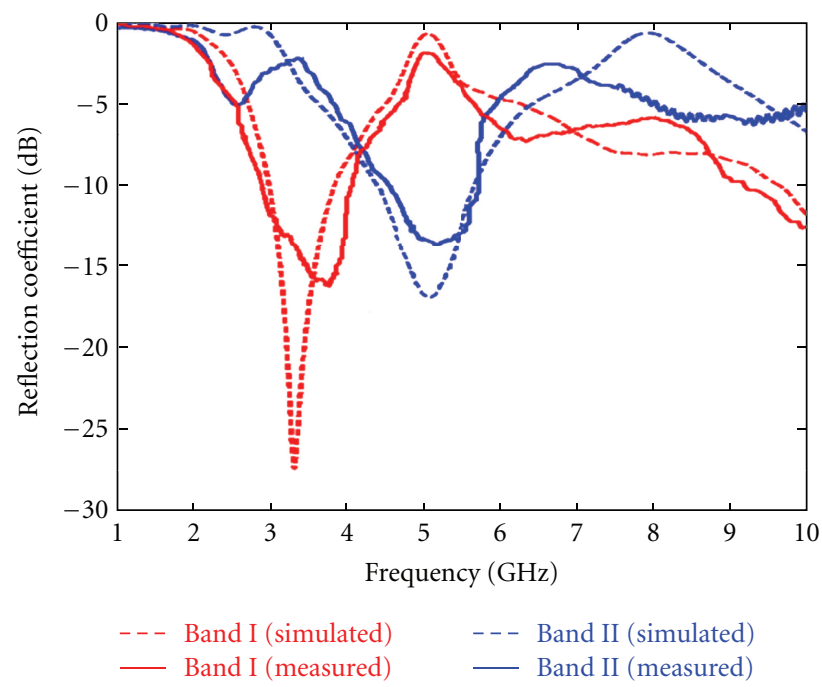

FIGURE 4: Simulated (dashed lines) and measured (solid lines) reflection coefficient curves when antenna is reconfigured in Band I: 3-4 GHz and Band II: 4.5-5.5 GHz.

\section{Simulation and Measurements Results}

3.1. Reflection Coefficient. All the simulations in this paper are performed using the CST microwave studio package which utilizes the finite integration technique for electromagnetic computation [9]. Figure 3 shows the simulated results for the reflection coefficient of the antenna when it is in the UWB mode and when it is notched around $5.8 \mathrm{GHz}$. Figure 4 depicts the simulated reflection coefficient when the antenna is configured to work into Band I and Band II. In general, a good agreement between simulated and measured results has been achieved. Small frequency shift $(<200 \mathrm{MHz})$ between the simulated and measured results has occurred; this can be accounted for by fabrication and material tolerances.
3.2. Surface Current. Figures 5(a) and 5(b) show the surface current distributions for the notched UWB antenna at $(3.4 \mathrm{GHz})$ in the passband and at the stopband $(5.8 \mathrm{GHz})$ respectively. At the stop band, the surface currents are condensed around the parasitic resonator and a strong coupling occurs (the direction of current flow on the resonator is opposite to the flow of the current on the nearby slots edges). Eventually, the surface current is largely reduced along the edges of the 2 slots and no radiation occurs, while in the pass band frequency $(3.4 \mathrm{GHz})$ the current flows smoothly on the edges of the slots without any perturbation by the resonator and hence radiation is as normal. Figure 6(a) shows the surface current distributions for the UWB antenna when it is operating in Band I at $(3.4 \mathrm{GHz})$ in the passband, and Figure 6(b) shows the surface current distributions for the 


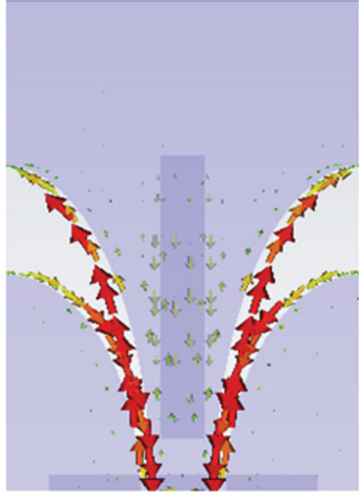

(a)
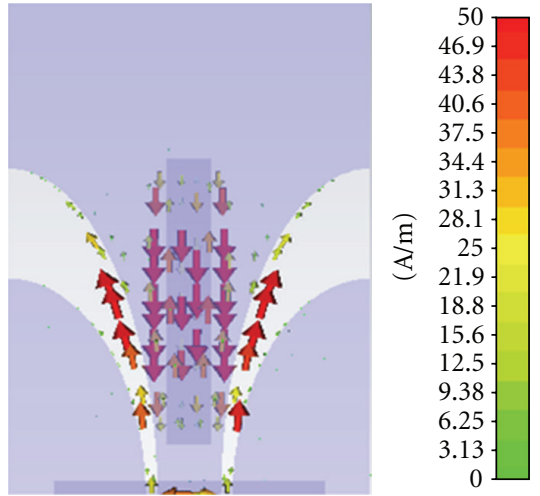

(b)

FIGURE 5: Simulated current distribution of the notched UWB antenna at (a) $3.4 \mathrm{GHz}$ (normal operation frequency) and (b) $5.8 \mathrm{GHz}$ (notched frequency).

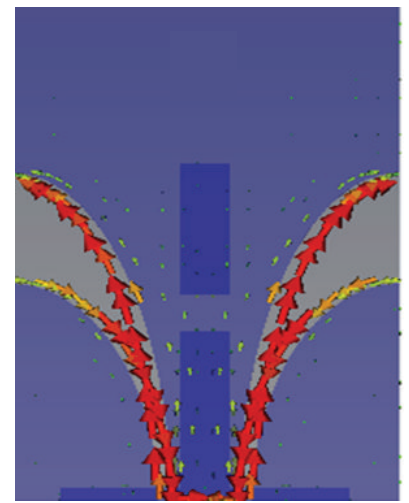

(a)

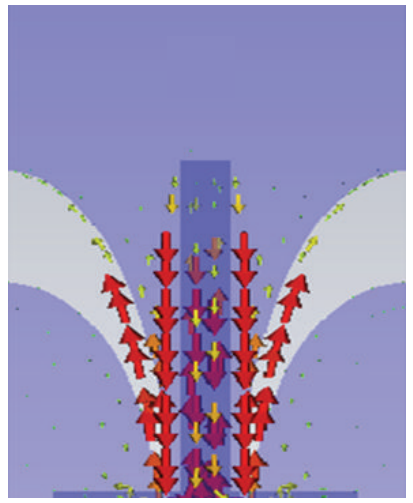

(c)
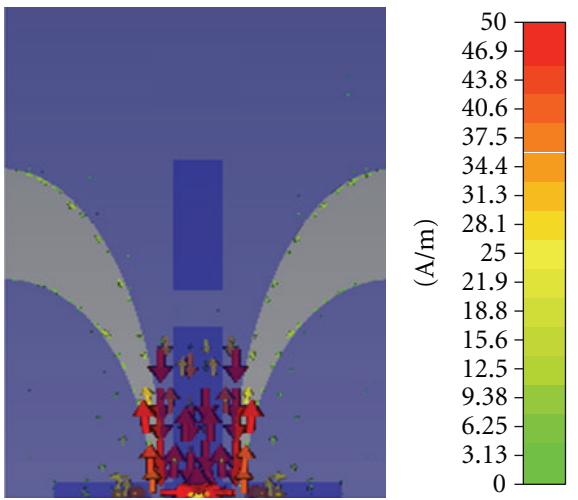

(b)
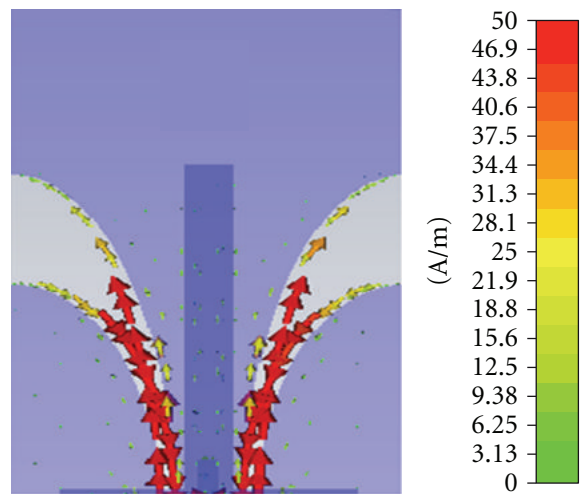

(d)

FIGURE 6: Simulated current distribution of the reconfigured UWB antenna in Band I: (a) $3.4 \mathrm{GHz}$, (b) $5 \mathrm{GHz}$, Band II: (c) $3.4 \mathrm{GHz}$, (d) $5 \mathrm{GHz}$.

UWB antenna when it is operating in the same band but at $5 \mathrm{GHz}$ which is out of band frequency, respectively. Figures 6(c) and 6(d) show the surface current distributions for the UWB antenna when it is operating in Band II at $(3.4 \mathrm{GHz})$ out of band frequency and Band II at $(5 \mathrm{GHz})$ which is in the passband, respectively. At the out of band frequency, the stub which is connected to the partial ground will change the input impedance of the antenna and the surface current will be more dominant around the stub while the surface current along the slots of the antenna is reduced. The effect of the stubs on matching the input impedance of the antenna can also be highlighted from Figure 7 which depicts the input impedance of the UWB antenna when it is reconfigured to work in Band I. For the UWB operation, the antenna input resistance $\left(R_{i}\right)$ should be around $50 \Omega$ and the input reactance $\left(X_{i}\right)$ should be close to $0 \Omega$ for the whole operating bandwidth. After reconfiguring the antenna to work into Band I, $R_{i}$ is close to $50 \Omega$ and $X_{i}$ is around $0 \Omega$ only at 


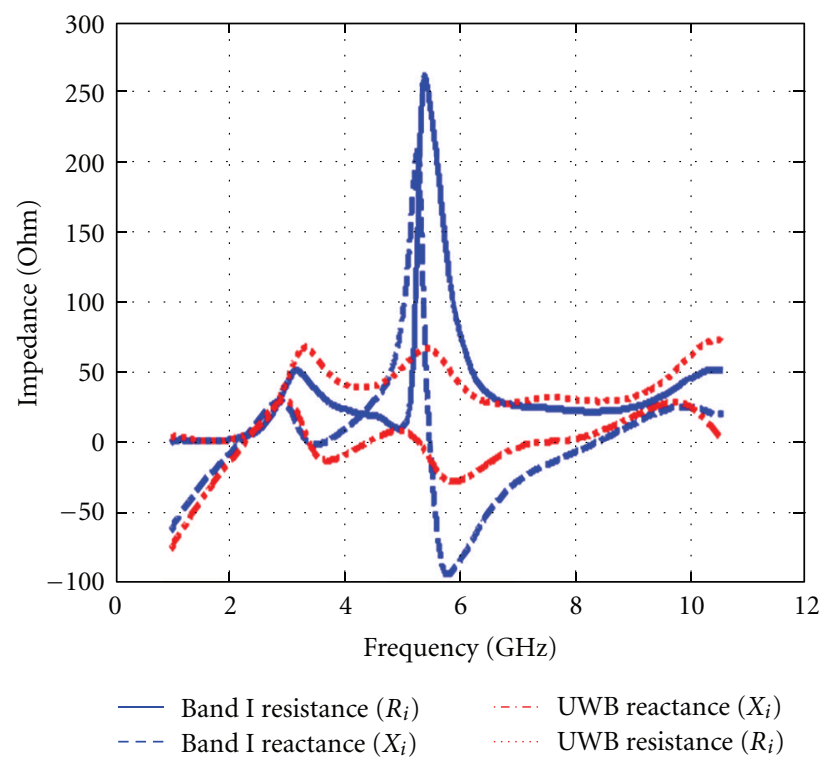

FIGURE 7: Simulated input impedance curves for the UWB antenna when the antenna is configured to operate in Band I.

TABLE 2: Simulated $(S)$ and measured $(M)$ realized gain.

\begin{tabular}{lccccccccc}
\hline \multirow{2}{*}{$\begin{array}{l}\text { Frequency } \\
(\mathrm{GHz})\end{array}$} & \multicolumn{10}{c}{ Gain (dBi) } \\
& $\mathrm{S}$ & $\mathrm{M}$ & $\mathrm{S}$ & $\mathrm{M}$ & $\mathrm{S}$ & $\mathrm{M}$ & $\mathrm{S}$ & $\mathrm{M}$ \\
\hline 3.4 & 1.9 & 2.4 & 1.9 & 2.5 & 2.1 & 3 & 0.8 & 0.7 \\
5 & 2.9 & 3 & 3.0 & 3 & -6.4 & -4 & 3.7 & 3.5 \\
5.8 & 3.6 & 3 & -7 & -4 & 1.9 & 2 & 3.8 & 3.7 \\
\hline
\end{tabular}

frequencies within the operating bandwidth (e.g., $3.4 \mathrm{GHz}$ ) and their values largely deviate from the nominal values outside the passband.

3.3. Radiation Patterns and Gain. The simulated and measured normalized radiation patterns at 3.4 and $5 \mathrm{GHz}$ for the UWB mode operation are plotted in Figures 8(a) and 8 (b), respectively. The simulated and measured normalized radiation patterns when the antenna is configured in Band I at 3.4 and in Band II at $5 \mathrm{GHz}$ are plotted in Figures 9(a) and 9(b), respectively. The $H$-plane patterns are omnidirectional and the E-plane patterns look like number " 8 " like a traditional monopole.

For radiation patterns measurements, the quality of the far field anechoic chamber is critically important. Slight misalignment uncertainties in the antenna under test (AUT) positioning can lead to discrepancies between simulated and measured radiation patterns. Moreover, the effects of the connectors and cables connected to the AUT were not simulated in the computational domain in spite of their capability to add some reflection in the chamber and deteriorate the perfect line-of-sight (LOS) assumption and add some ripples on the radiation patterns.

It is worth mentioning here that the radiation patterns did not change when we reconfigure from the UWB operation to the reconfigured cases. Table 2 summarizes a comparison of the simulated and measured realized peak
TABle 3: Measured total efficiency.

\begin{tabular}{lcccc}
\hline $\begin{array}{l}\text { Frequency } \\
(\mathrm{GHz})\end{array}$ & UWB & Notched UWB & Band I & Band II \\
\hline 3.4 & 87 & 88 & 90 & 40 \\
5 & 90 & 87 & 30 & 91 \\
5.8 & 85 & 45 & 55 & 88 \\
\hline
\end{tabular}

gain between the UWB case, the notched UWB, and the 2 reconfigured cases. At $5.8 \mathrm{GHz}$ (notch frequency), the UWB notched antenna showed hugely reduced broadside gain compared to the UWB case and the reconfigured cases show slight increase in peak gain for passband frequencies compared to the UWB case.

3.4. Efficiency. Radiation efficiency is the ratio of input power that ends up as radiated power to the net power accepted by the antenna and the total efficiency is the ratio of the total power radiated from the antenna to the total net power applied at the antenna input terminal. Total efficiency results usually give better indication than return loss results to highlight the filtering performance of the antenna; this is because the antenna total efficiency not only accounts for the input mismatch of the antenna but also accounts for the ohmic losses in the antenna. Figure 10 depicts the simulated values of the total efficiency, when the antenna is operating in the UWB mode and the notched UWB mode and when the antenna is configured to work in Band I and Band II. The total efficiency was measured using the modified wheeler cap methods as in [10-12]. Table 3 shows the measured total efficiency when the antenna is operating in the UWB mode and the notched UWB mode and when the antenna is configured to work in Band I and Band II. The measured values agree well with the simulated ones. From Figure 10 and Table 3 we can see how poor the total efficiency gets at 

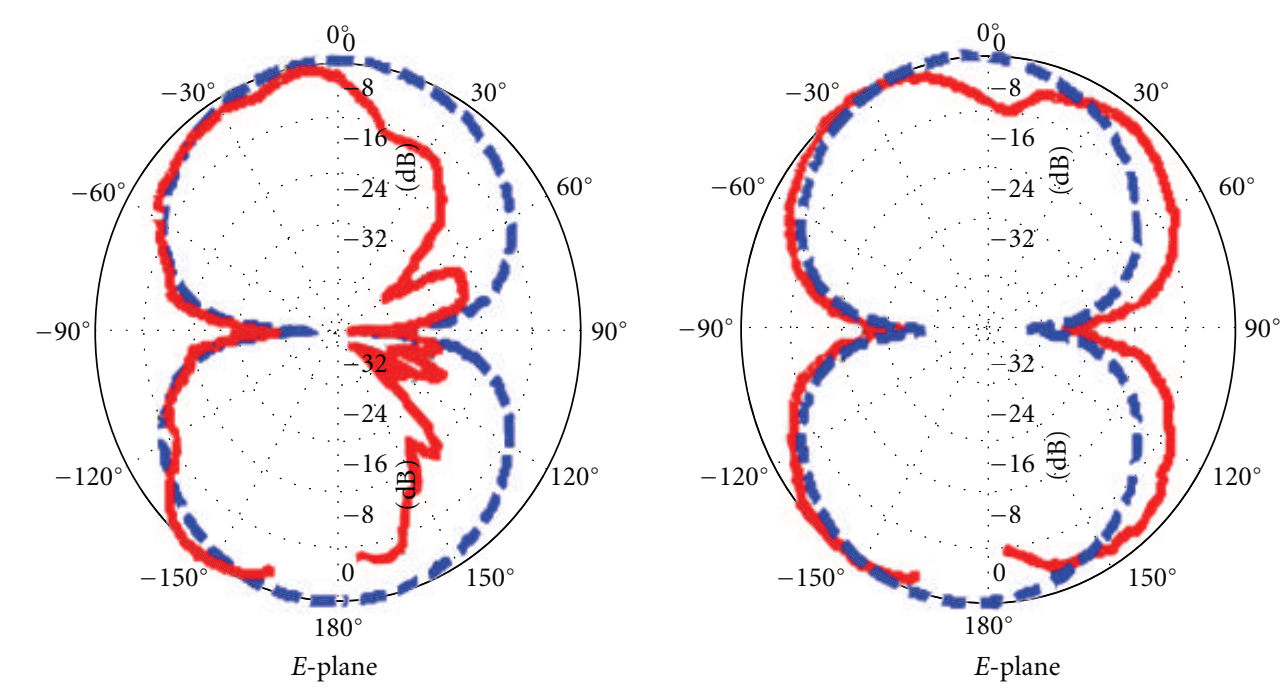

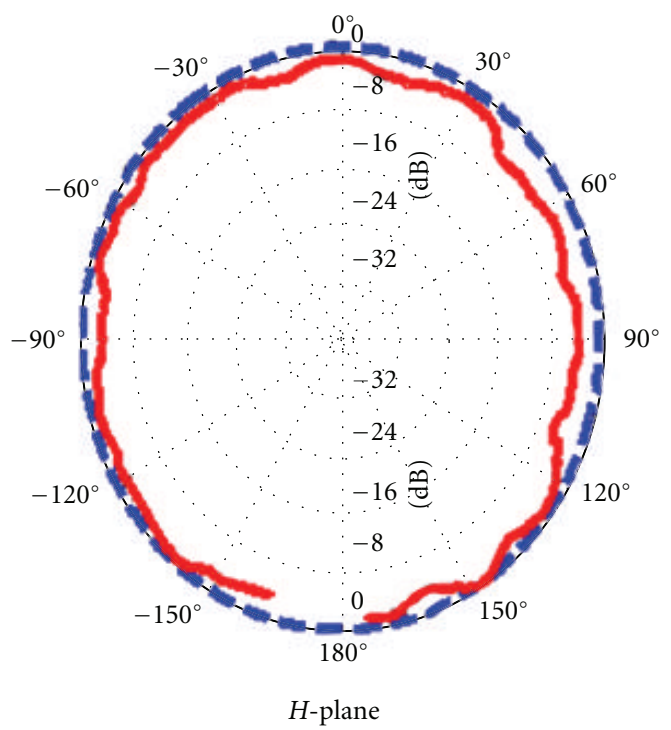

(a)

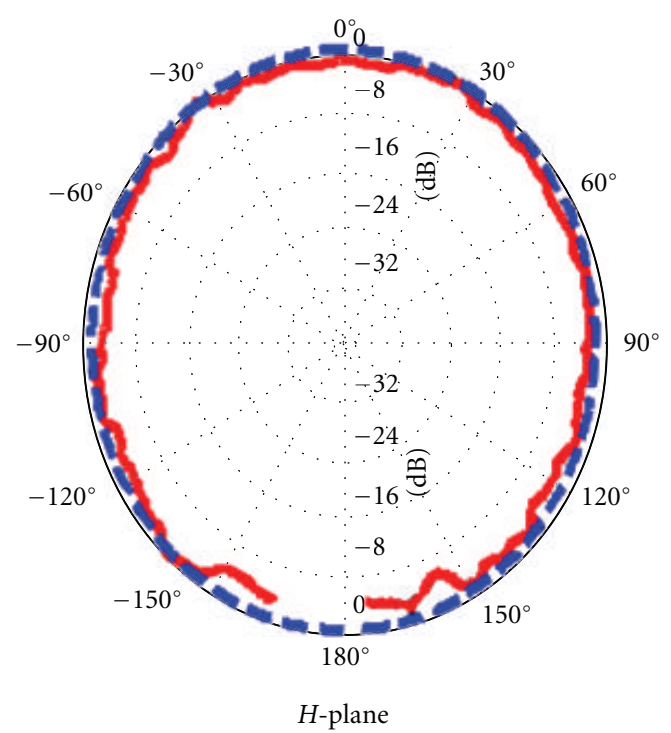

(b)

FIgURE 8: Simulated (dashed blue line) and measured (solid red line) normalised $E$-plane $(X-Y)$ and $H$-plane $(X-Z)$ radiation patterns, when antenna is in the UWB mode: (a) $3.4 \mathrm{GHz}$ and (b) $5 \mathrm{GHz}$.

the notch frequency in UWB notched case or at out-of-band frequencies in the reconfigured bands I and II.

\section{Applicability to Cognitive Radio}

The previously discussed antenna can be easily integrated into cognitive radio; when the antenna is operating in the UWB mode, it can sense the whole spectrum to find the spectrum holes and then from sensing information it will decide to switch to work in one of the available predefined bands (e.g., Band I or II). Usually sensing places severe requirements on sensitivity, linearity, and dynamic range of the cognitive radio RF front end. This is because the $\mathrm{RF}$ signal presented at the antenna of a cognitive radio includes signals from close or separated transmitters and from transmitters operating at different power levels [13]. Detection of weak signals might frequently be performed in the presence of very strong signals. Thus, to relax the strict requirements placed on the RF analogue circuits, it would be beneficial from a system point of view if we can mitigate an interferer before it saturate our RF front end. This can be done by using the UWB notched mode during sensing. It is worth mentioning here that not only this notched UWB mode can be good for sensing, but also it can be helpful in communication between cognitive secondary users who can still communicate more efficiently using the notched UWB channel (e.g., more allowed transmitted power and longer transmission range with higher data rate) without affecting or being affected by a specific legacy primary user who is currently operating at the notched frequency band.

\section{Conclusion}

A reconfigurable UWB tapered slot antenna (TSA) has been demonstrated and reconfigurability is introduced by 

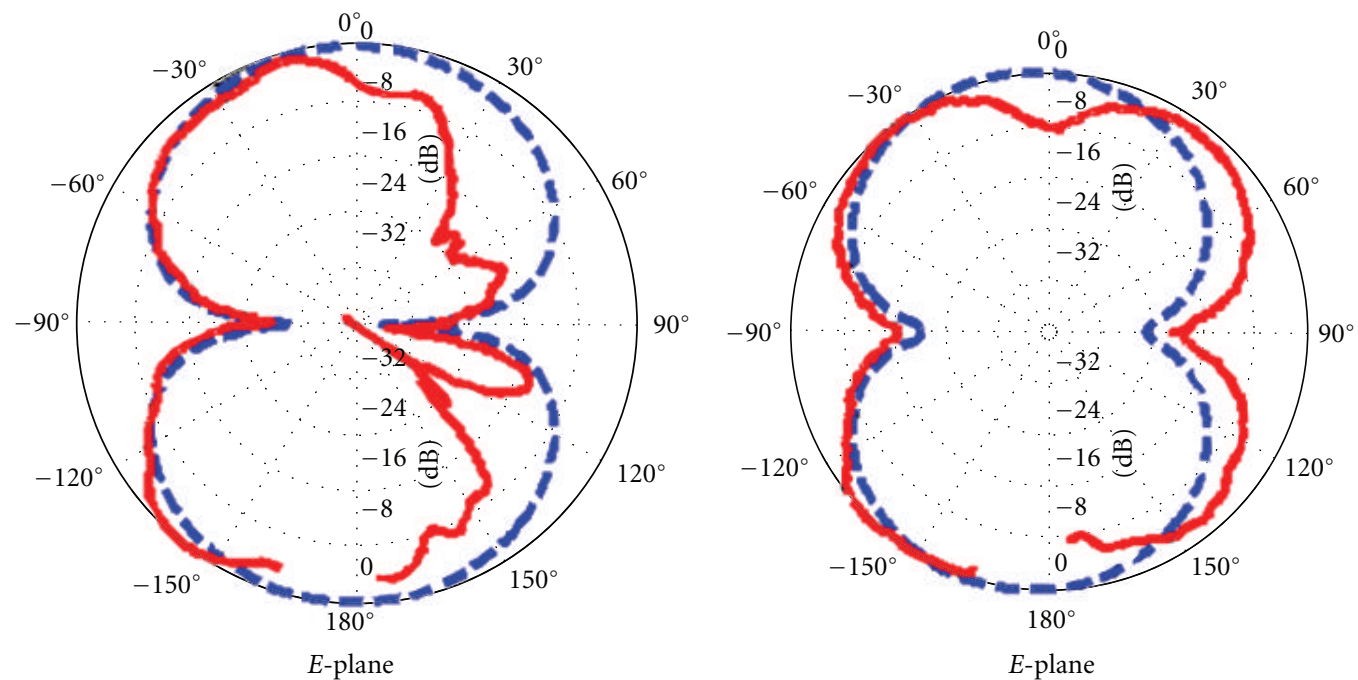

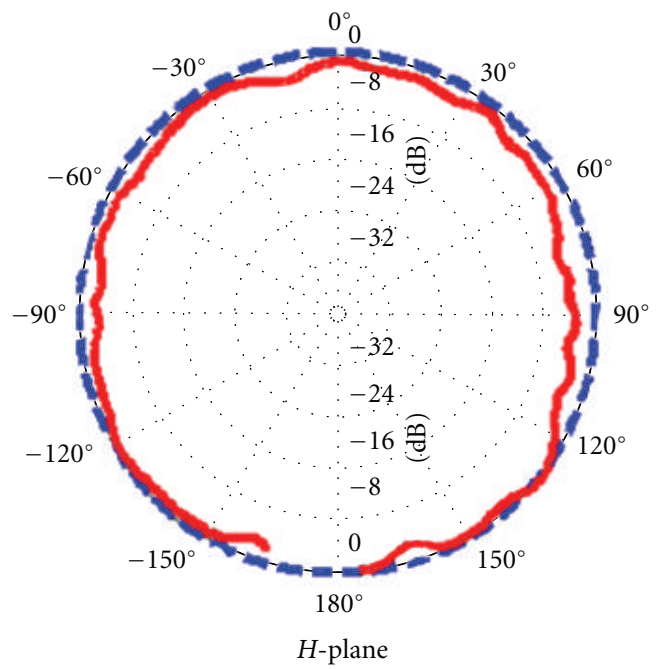

(a)

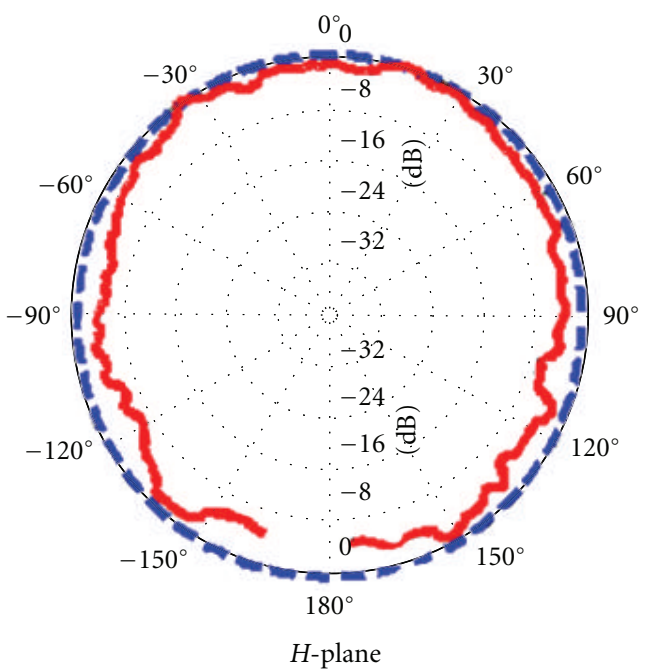

(b)

FIGURE 9: Simulated (dashed blue line) and measured (solid red line) normalised $E$-plane $(X-Y)$ and $H$-plane $(X-Z)$ radiation patterns, when the antenna is configured at: (a) $3.4 \mathrm{GHz}$ in Band I and (b) $5 \mathrm{GHz}$ in Band II.

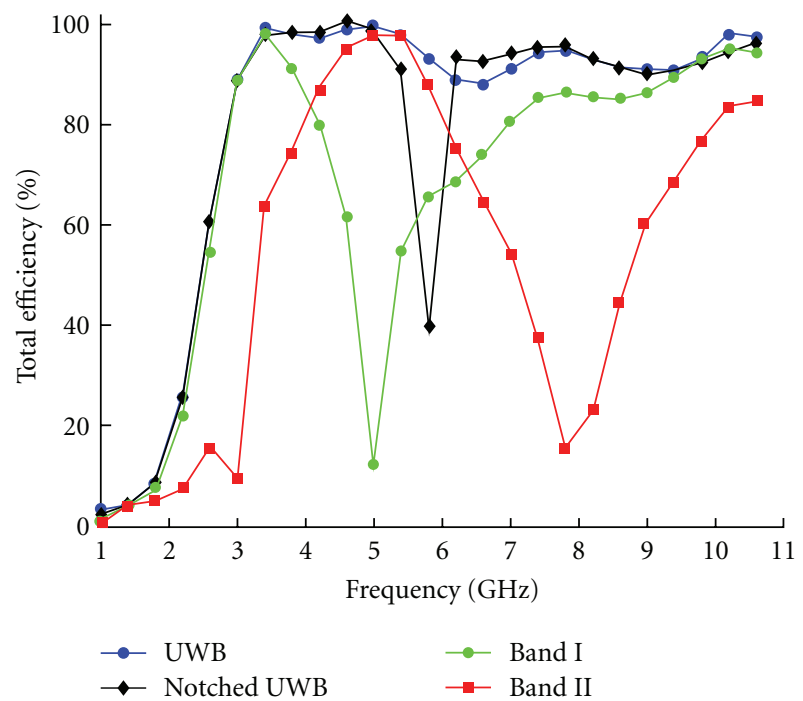

FIGURE 10: Simulated total efficiency when the antenna is operating in different modes. 
varying the length of a stub connected to an additional tiny partial ground on the back of the antenna. The proposed topology is versatile in terms of the availability of different reconfiguration bands and the antenna is very compact and simple. Another interesting feature of the antenna is that this ground stub can be used as a parasitic element electrically coupled to the TSA antenna to notch the UWB operation at a specific frequency. The simulated and measured reflection coefficient, radiation patterns, and gain for the UWB mode, the notched UWB mode, and the reconfigured Bands I and II were presented and they all showed a very good performance. Applications requiring frequency band switching such as cognitive radio could benefit from the proposed reconfigurable TSA antenna as we can use the UWB mode or the notched UWB mode in sensing and the reconfigured bands for communication purpose.

\section{References}

[1] J. R. Kelly, P. S. Hall, and P. Gardner, "Integrated wide-narrow band antenna for switched operation," in Proceedings of the 3rd European Conference on Antennas and Propagation (EuCAP '09), pp. 3757-3760, March 2009.

[2] T. Aboufoul and A. Alomainy, "Reconfigurable printed UWB circular disc monopole antenna," in Proceedings of the Antennas \& Propagation Conference (LAPC '11), pp. 1-4, Loughborough, UK, November 2011.

[3] T. Aboufoul and A. Alomainy, "Single-element reconfigurable planar ultra wideband antenna for cognitive radio front end," in Proceedings of the 4th International Conference on Cognitive Radio and Advanced Spectrum Management (CogART '11), Barcelona, Spain, October 2011.

[4] T. Aboufoul, A. Alomainy, and C. Parini, "Reconfiguring UWB monopole antenna for cognitive radio applications using GaAs FET switches," IEEE Antennas and Wireless Propagation Letters, vol. 11, pp. 392-394, 2012.

[5] X. Artiga, J. Perruisseau-Carrier, P. Pardo-Carrera, I. LlamasGarro, and Z. Brito-Brito, "Halved Vivaldi antenna with reconfigurable band rejection," IEEE Antennas and Wireless Propagation Letters, vol. 10, pp. 56-58, 2011.

[6] J. Perruisseau-Carrier, P. Pardo-Carrera, and P. Miskovsky, "Modeling, design and characterization of a very wideband slot antenna with reconfigurable band rejection," IEEE Transactions on Antennas and Propagation, vol. 58, no. 7, pp. 22182226, 2010.

[7] A. Rahman and Y. Hao, "A novel tapered slot CPW-fed antenna for ultra-wideband applications and its on/offbody performance," in Proceedings of the IEEE International Workshop on Antenna Technology (iWAT '07), pp. 503-506, Cambridge, UK, March 2007.

[8] Advanced Design System (ADS), “Agilent Technologies," Santa Clara, Calif, USA, 2005, http://www.agilent.com.

[9] CST-Microwave Studio, “User's Manual," 2011.

[10] H. A. Wheeler, "The radian sphere around a small antenna," in Proceedings American Institute of Electrical Engineers (IRE '59), vol. 47, pp. 1325-1331, August 1959.

[11] H. G. Schantz, "Measurement of UWB antenna efficiency," in Proceedings of the IEEE VTS 53rd Vehicular Technology Conference (VTS SPRING '01), vol. 2, pp. 1189-1191, May 2001.

[12] M.-C. Huynh, Wideband compact antennas for wireless communication [Ph.D. dissertation], Department of Electrical and Computer Engineering, Virginia Polytechnic Institute, 2004.
[13] D. Cabric, S. M. Mishra, and R. W. Brodersen, "Implementation issues in spectrum sensing for cognitive radios," in Proceedings of the Conference Record of the 38th Asilomar Conference on Signals, Systems and Computers, vol. 1, pp. 772776, November 2004. 

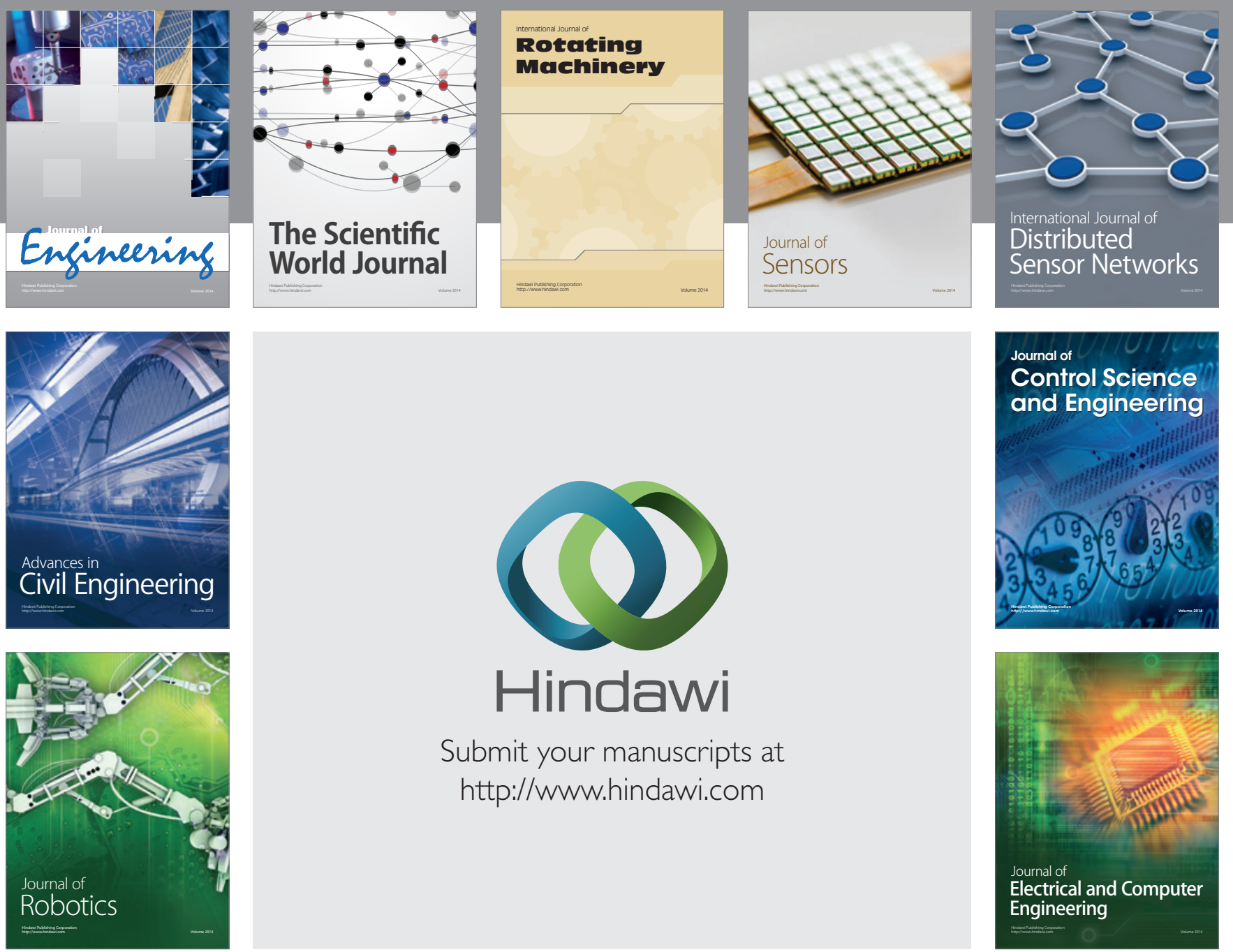

Submit your manuscripts at

http://www.hindawi.com
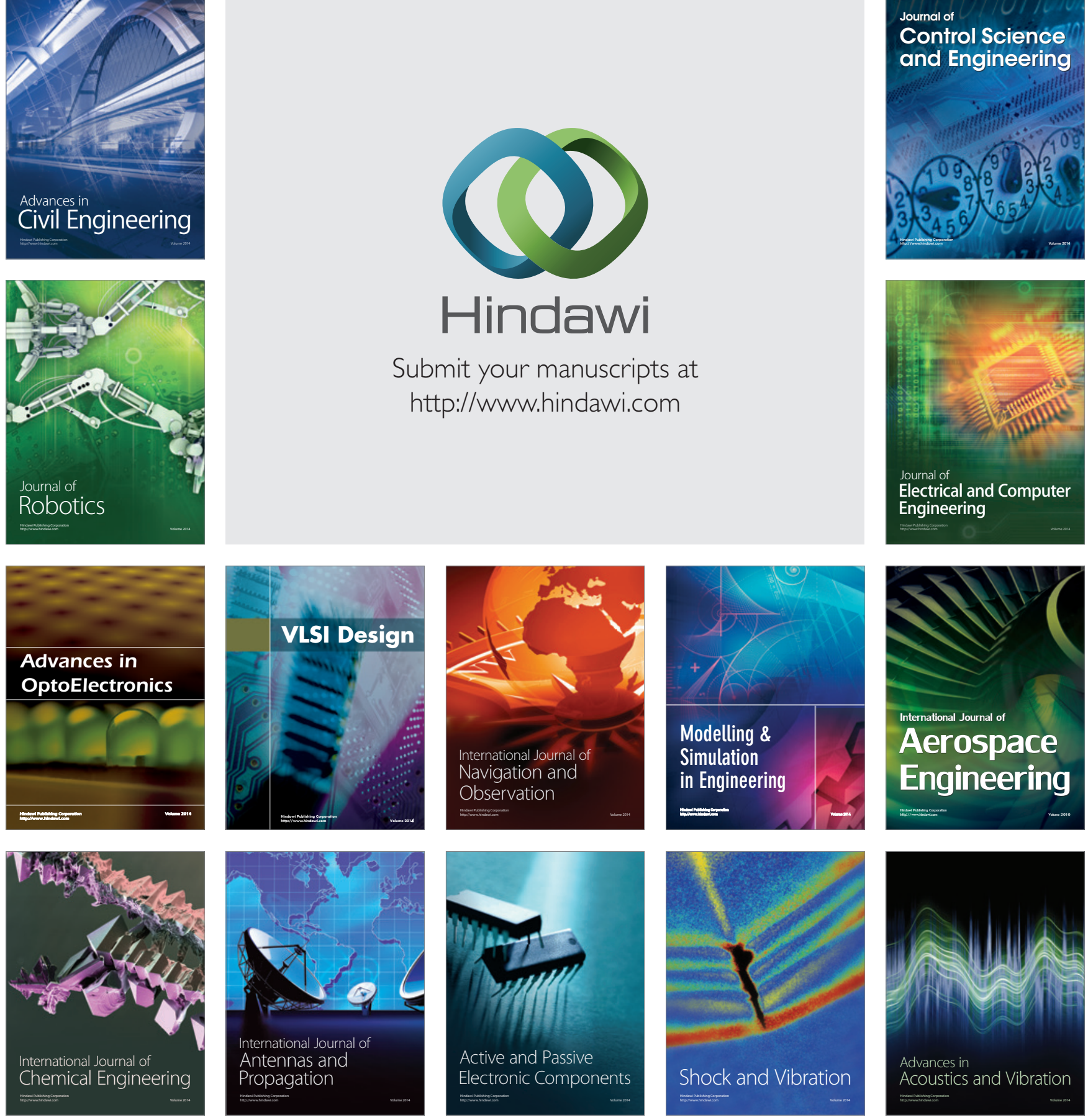